\title{
El Negocio de Líneas de Cruceros y el Modelo de Estrategias Competitivas
}

\author{
Maria T. Zancudo G.' \\ Guillermo Alvarez ${ }^{2}$
}

RESUMEN: En este trabajo se plantea la aplicación del modelo de estrategias competitivas de Michael Porteren la industria de la hospitalidad, fundamentalmente en el negociodelas lineas de cruceros. La metodologíautilizada es la de estudio de casos, especificamente la línea de cruceros Carnival, Inc., la cual es la empresa líder en el negocio de cruceros en Americadel Nortey la más grande del mundo en ténninos de transporte de pasajeros. Los resultados obtenidos evidencian que la utilización del modelo de estrategias competitivas de Porter, podria convertirse en un enfoque gerencial que optimice a las organizaciones de la industria de la hospitalidad.

PALABRAS CLAVES: Industria de turismo; líneas de cruceros; competitividad; modelode estrategias de Porter; Canival.

ABSTRACT: This work describes the application of Michael Porter'stheoryon competitivestrategytoanalize the hospitality industry: caseof Camival Crnise Line, Inc., which is the leading company in the cruise ship business in North America, and the world largest cruise line in

1. Bachelor en Administración de Hoteles y Restaurantes, University of Denver. Postgraduado en Educación en la Universidad Pedagógica Experimental Libertador, Venezuela, y en Administración de Hoteles y Restaurantes en Florida Intemational University. Profesor de la Universidad Simón Bolívar, sede del Litoral, en el área de Turismo y Hotelaria.

End. para corresp.: Universidad Simón Bolívar, sede del Litoral; Camuri Grande, Apartado Postal 314, La Guaira, Municipio Vargas, Distrito Federal, (031) 722911 al 15, ext. 245, 188, 152.

2. Postgraduado en Educación Superior Universitaria, en Psicologia y en Ingeniería Empresarial de la Universidad Simón Bolivar. Profesor de la Universidad Simón Bolivar en el área de Psicologia Organizacional y de Gerencia de la Empresa.

End. para corresp.: Universidad Simón Bolívar, sede del Litoral; Camuri Grande, Apartado Postal 314, La Guaira, Municipio Vargas, Distrito Federal, (031) 722911 al 15, ext. 245, 188, 152. 
termis of passengers count. Results demostrate that organizations can create and sustain a competitive advantage in its industry and also that Porter's theory advantage in its industry and also that Porter's theory
could beapowerful management tool tooptimize hospitality industryorganizations.

KEY WORDS: Tourisn industry; cruise lines competitiveness; Porter'smodel on competitive strategy: Cannival.

\section{Introducción}

Las organizaciones funcionan en un ambiente de permanente interacción entre unas y otras: dondelo económico, técnico, social y político están en constantes cambios A esto se le conoce como mercados competitivos. Para que una organización alcance y mantenga una posición competitiva en este ambiente, necesita definir y desarrollar una estrategia; sabiendo que las fuerzas interactivas, internas y externas al mercado, afectan las posibles estrategias a seguir y por ende los resultados de su aplicación.

De acuerdo a Porter (1985) para que una organización se conviertaen exitosa, necesitaubicarse en una posición donde identifique alguna ventajacompetitiva. Para ayudar a las organizaciones en este proceso, desarrolló un modelo - Modelo de Estrategias Competitivas - donde describe técnicas tanto para análisis de la industriay sus competidores, como para la creacióny el mantenimiento deesa ventaja competitiva. La metodología permite identificar una posición competitiva favorable a partir del análisis de cinco factores - las fuerzas competitivas-, cuya interacción dan forma a la estructura de cualquier industria, a saber:

- el riesgo para entrar a la industria;

- el poder de los compradores.

- el riesgo a ser sustituido

- el poder de los distribuidores;

- la rivalidad entre los competidores existentes.

Adicionalmente, estas fuerzas determinan dos hechos importantes:

- lo atractivo de las ganancias a largo plazo para una industria;

- la naturaleza de la competencia en esa industria.

Para aplicar el modelo de estratégias competitivas es necesario seguir los siguientes pasos:

- identificar el ambiente competitivo de la industria a través del análisis de interacción de las cinco fuerzas competitivas así como la habilidad de cada una de ellas para influir la productividad y rentabilidad de la industria;
- establecer el posicionamiento de la empresa en ese ambiente

- desarrollar las alternativas estratégicas de la organización.

Laesencia de formular una estrategia competitiva se refiere a la necesidad de optimizar la relación de una empresa con su medio ambiente. En general, la industria dela hospitalidad se caracteriza por ser una industria de alto riesgo debidoal intenso requerimiento de capital y recurso humano: los gastos de personal y el índice de rotación son muy altos; y, se requiere de una gran inversión para poseer una unidad de producción sea propia o rentada. Las líneas de cruceros constituyen un segmento en la industria de la hospitalidad, que en los últimos años ha mostrado un rápido crecimiento. Entre los indicadores se destacan:

- un incremento del $9.9 \%$ anual de las tasas de reservaciones de cruceros a nivel mundial(Roccoy Andrew, 1991):

- laincorporación de 34.315 embarcaciones, durante losaños del 1984 al 1990, a las 41.000 que había en el mercado en 1983, y la proyección en un 39\% de la capacidad de crecimiento para la década de los 90 's.

- la ampliación y diversificación de los segmentos de mercado a medida que los cruceros han sido capaces de atraer el mayor grupo de personas jovenes: de acuerdo a la Asociación Internacional de Líneas de Cruceros - CLIA (1989), 30\% delos pasajeros de los cruceros tienen edades comprendidas entre los 25 y 30 años, y $58 \%$ tienen menos de 60 años, lo que significa que la edad promedio de estos pasajeros se encuentra en el rango entre los 40 y 59 años;

- representa el porcentaje más alto en el negocio de viajes, que el de cualquier otro segmento, y es a la vez el renglón más rentable que losagentespuedenvender: entre el $95 \%$ y $99 \%$ de las ventas de cruceros se realiza a través de las agencias de viajes (Dickenson, 1990).

Del escenarioplanteado sederiva lo relevante que resultaría analizaren detalle las características del negocio de las líneas de cruceros como empresas inmersas en un medio fuertemente competitivo. Por otra parte, la existencia de una herramienta gerencial como la que plantea Michael Porter, proporciona la oportunidad de analizar con criterios científicos la posición competitiva actual y prospectiva de una empresa.

En este contexto, el problema a estudiar se formuló de la siguiente manera: ¿Cuáles son las características del entorno competitivo donde funcionan las líneas de cruceros ?; ¿Cuál es el posicionamiento de la empresa Carnival Cruise ?; ¿Cuál es la estratégia competitiva que sigue la empresa?

Con el presente estudio se aspira a responder a todas y cada una de las interrogantes propuestas. Igualmente se propone como objetivo general aplicar el modelo de estratégias competitivas a laslíneas de cruceros, en particular a la empresa Carnival Cruise; y como objetivos específicos:

identificar las características del entorno competitivo donde funcionan las líneas de cruceros: 
- determinar el posicionamicnto de la cmpresa Carnival Cruise;

- identificar la estratégia competitiva que sigue la compresa

\section{Metodologia}

Este trabajo es un estudio de caso, con base documental (Barrioset al., 1987). Se pretende identificar la estratcgia competitiva que utiliza una empresa con évito, utilizando las etapas que se plantean en el modelo de estrategias competitivas de Porter.

Se cumplió con el siguiente esquema de trabajo, a saber:

- identificación del ambiente competitivo de la industria a través del análisis de la interacción de las cinco fucrzas competilivas así como la habilidad de cada una de cllas para influir la productividad y rentabilidad de la indust ria;

- determinación del posicionamiento de la empresa cn ese ambiente;

- identificación de las alternativas estratégicas de la organización.

\section{Resultados}

Con base al análisis de la interacción de las cinco fuerzas competitivas, se puede derivar los siguientes resultados:

\section{Ambiente Competitivo de las Lineas de Cruceros}

El ambiente competitivo para las líncas de cruceros se muestra atractivo, ya

- siendo un segmento de la indust ria de la hospitalidad, la est ruclura competitiva de las líneas de cruceros es similar a la de ésla, a saber: a) son empresas de alto riesgo debido al intenso requerimiento de capital y recurso humano que se requiere para poseer una unidad de producción, y b) su mercado, especialmente en los paises más desarrollados, se considera bastante concurrido, diversificado y fuertemente competido. Esto ha traído como consecuencia, un desbalance entre la oferta y la demanda, lo cual fortalece el poder y capacidad de negociación de los consumidores y la oferta de productos sustitutos. Sin embargo, cabe destacar que las líneas de cruceros cuentan con mayor fucrza competitiva que el resto de los segmentos de la industria de la hospitalidad

- los ricsgos que deben confrontar las nuevas empresas al incorporarse a la indusiria, es una fuerza que favorece a las lincas de cruceros que ya están en el mercado. Las barreras para losque pretenden iniciar son grandes y la principal se refiere a los requerimientos de capital ya que se requiere una gran inversión funanciera no sólo para la adquisición de los buques (unidades de producción), sino también para la publicidad y promoción (inversión ricsgosa y no recuperable), así como también, un considerable capital de trabajo. La industria requiere de un ingreso a gran escala y esto condiciona a los nuevos inversores o cmpresarios, pues lo contrario representa una desventaja desde el punto de visia de costos y por lo tanto competitiva. Igualmente. el acceso a los canales de distribución representa una barrera para incorporarse como línea de crucero. Los agentes de viajes deben estar convencidos de la seguridad y calidad del muevo servicio que ofrecen frente a lo que ofrecen las líncas establecidas. Esto no es tarea fácil, por el contrario se requicre de un esfuerzo de venta agresivo, costoso e intenso;

- la tasa de crecimiento del negocio se ha incrementado durante las dos últimas décadas y se espera que continúe creciendo. El mercado en este aspecto es muy particular en términos de balance. Una sola línea capta un cuarto del total del mercado norteamericano y ransporta cl doble de los pasajeros que su competidor máscercano (Camival Cruise Line, 1991:4). De acuerdo al modelo de Porter (1980), un líderde esta magnitud impone disciplina en el mercado asi como también juega un rol de coordinador en la indust ria a través de cstratcgias como el liderazgo en precios (esteexactamente el caso de la línea de cruceros Carnival). Entre las otras firmas existe la rivalidad para tratar de ampliar su porción en el mercado

- el producio sustituto más importante para los cruceros son los resorts y los clubes que ofrecen todos los servicios en una misma localidad. Sin embargo, a nivel internacional las líneas de cruceros mantienen su mercado. Algunos analistas financicros aseguran que este crecimiento continuará y aumentará, a pesar del despliege de los clubes de ticrra(Roccoy Andreiv, 1991). Por lo tanto, no se percibe un riesgo intenso desde esta fuente. Sin embargo, para responder a ésta amenaza, las líneas de cruceros, ofrecen a los consumidores paqueles combinando los cruceros con porciones terresires;

- el poder de los consumidores de las líneas de cruceros proviene de las agencias de viajes, ya que cl $99 \%$ de los cruceros se venden a través de ellas. Esto hace a las líneas de cruceros débiles frente al poderde los consumidores ya que dependen de un solo canal de distribución. Por su parte los agentes de viajes influyen directamente el proceso de compra de los consumidores dado que son ellos quienes conocen las caractcrísticas y detalles del producto. Estc factor hace a la industria vulnerable y gran parte de las ganancias escapan de su control;

- el poder de negociación de los provecdores no representa una gran amenaza para las líneas de cruceros. Por el contrario, ellas se benefician de su naturaleza de industria global para sobre ponerse a cualquier ricsgo intenso que pudiera originarse de los provecdores. Actualmente, la mayoría de los barcos tienen sus proveedores distribuidos a lo largo de las rutas de sus cruceros. Sin cmbargo, cs importante señalar dos factores que pudieran cjercer un presión circunstancial, 
estos son: el combustible y la fuerza laboral. Porter se refierc al recurso humano como un provecdorpoderosoya queel personal altamente calificadoy/o fuertemente sindicalizado puede a través de la negociación obtener una parte significativa o las ganancias potcnciales de una cmpresa. El recurso humanopuede ser unafuente importante de presión para todas las empresas de servicio, las cuales por naturaleza tienen una intensa fuerza laboral.

\section{Posicionamiento de la Línea de Cruceros Carnival, Inc.}

En base al número de pasageros transportados, Carnival es la línea de cruceros más grande del mundo. Fue fundada por Ted Arison en 1974 y registrada como una corporación panameîa c inició sus operaciones con un solo barco. Hoy en día la línea de cruceros Carnival csta integrada por nucve unidades de cruceros y otras propiedades: el resort y casino Carnival CristalPalace en Nassau, Bahamas; la línea de cruceros Holland America, la cual cuenta con cuatrobarcos de cruceros que navegan en Alaska y el mar Caribe; la cadena de hoteles Westmart, la cual está integrada por diecinucve hoteles; la cmpresa Westour que es el mayorista de turismo más grande de Alaska y cl oeste canadiense; y finalmente, la línea de cruceros Winstar cuya flota de tres barcos, son los de mayor capacidad de pasajeros en el mundo. Así también, estableció una compañía con la operadora de hoteles Continental, para administrar el Cristal Palacc. Entre sus recientes adquisiciones (1992-1994) se encuentran dos ultra lujosos barcos de cruceros de diseño singular y cficiente operación (Carnival Cruise Line, 1994) y en Venezuela (1994)el Hotel Marcma re Resort.

\section{Estrategia Competitiva de la Linea de Cruceros Carnival}

De acucrdo con Porter (1985), una estrategia de liderazgo en cuantoa costos, en algunas oportunidades puede revolucionar una industria en la cual historicamente, la competitividad se ha basado en criterios diferentes a los de costos y precios. En este sentido, los competidores adolecen de una adecuada preparación económica o perceptiva para tomar las acciones necesarias para minimizar sus costos. Esto refleja la razón de las ventajascompetitivas de Carnival en la industria de hospitalidad.

La posición de Carnival en la cstructura de la industria de la hospitalidad puede ser considerada como muy poderosa. Carnival es una corporación global, integrada por diferentes compañías con una diversidad de recursos y lo más importante, con una gran capacidad de enfrentar la competencia (competitividad).

Carnival ha desarrollado con mucho éxito una estrategia de liderazgo en cuanto a costos. Esto se deriva ya que no sólo ha hecho frente y ha mantenido su posición ante poderosos compradores, sino que ha asumido una actitud agresiva para infuenciar a su favor el mercado y cn consecuencia el balance de la estructura de la industria.
Desde sus inicios Carnival comenzó innovando y con un buen producto. Cuando los cruceros eran considerados como el tiempo y lugar ideal para descansa y mantenerse tranquilo de una manera pasiva, la línea de cruceros Carnival introdujo "el crucero de la diversión", creando de ésta manera un simbolo para su producto

En 1978, cuando los costos de construcción de barcos alcanzaron altos niveles de record y el precio de los combustibles amenazaron con minimizar el concepto de vacaciones en el mar, Ted Arison anunció que Carnival construiría un barco nuevo. Muchos expertos pensaron que sería una unidad pequeña, con la capacidad de pasajeros justa que permita exceder por poco los gastos de operación para así poder ofrecer precios competitivos en el mercado actual (Carnival Cruise Leine, 1991). Sinembargo, lasideas de Arisoneran que sería economicamente rentable construir nuevos barcos de mayor capacidad, usando todos los avances posibles en la tecnología, con un diseño

la vez mantener los costos a un nivel real. Desde entonces, los criterios prevalentes en la industria son espacio, confort y eficiencia (Carnival Cruise Leine, 1991).

Por otra partc, la línea decruceros Carnival ha obtenidoimportantes ventajas en cuanto a los costos de operación, derivadas de ser una corporación que integra varios sectores de una misma industria, lo cual lepermite un mejor control del producto que ofrece. La posibilidad de combinar vacaciones en el marcon porciones terrestres favorece a Carnival para ofrecer un producto a mejor precio y calidad y por tanto, obtener una mayor porción del mercado.

Carnival inició sus opcraciones con una estrategia de diferenciación luego alcanzó su liderazgo en la indust ria ofreciendo un producto a buen precio y percibido comode excelente valor. Hoy en día, Carnival promociona sus precios competitivos usando su simbolo por el cual ya es reconocido los barcos de la diversión ("the fun ships"). Después de diecisiete años, la continuidad en la gerencia se perfila como una gran fortaleza para que la línea de cruceros Carnival alcanzara y mantuviera una creciente organización rentable y productiva que ofrece beneficios a sus consumidores aún no superados por sus competidores.

Las organizaciones funcionan en un ambiente de permanente contacto (interacción) entreunas y otrasenel cual los resultadosdelas estrategias organizacionales dependen de lo que hagan otras organizaciones. Para que una organización alcance y mantenga una posición en estc ambiente, necesita desarrollar una estrategia. Fuerzas interactivas internas y externas al mercado afectan las posiblesestrategias a seguir y por ende los resultados de esas escogencias (alternativas). De acuerdo a Michael Porter, la mejor alternativa de una organización exitosa es ubicarseen la posición donde identifique alguna ventaja competitiva en su industria.

\section{Conclusión}

Las empresas del sector económico de servicios enfrentan situaciones administrativas que no son comunes en las empresas manufactureras. Por ello es necesario establecer y aplicar técnicas administrativas que sean capaces de dar 
cabida tanto a los principios básicos universales como a las necesidades particulares de los gerentes de estas organizaciones.

Analizar en detalle las características del negocio de las líneas de cruceros, fundamentalmente las de Carnival Cruise, utilizando la herramienta gerencial que plantea Michael Porter, ha proporcionado la oportunidad de fundamentar con criterios científicos la posición competitiva actual y prospectiva de una empresa.

Losresultados obtenidosevidencianque la utilización del modelo de estrategias competitivas de Porter, podría convertirse en un enfoque gerencial que optimice a las organizaciones de la industria de la hospitalidad.

\section{Referencias Bibliográficas}

BARRIOS et al. 1987. Manual del trabajo de grado para el título de magister. Caracas: Universidad Pedagógica Experimental Libertador.

CARNIVAL Cruise Line. 1991. Crwise News: Seventeen Years of Success. Miami, U.S.A.

CRUISE LINES International Association. 1989. Highlights 1989 CLIA cruise market profile study. New York. DICKENSON, B. 1990. Travel Weekly: Love Affair with Agents. July, 16.

PORTER, M. 1980. Competitive strategy: techniques for analizing industries and competitors. New York: The Free Press a division of Macmillan, Inc.

1985. Competitive advantage: creating and sustaining superior performance. New York: The Free Press a division of Macmillan, Inc.

ROCC:O, A. y ANDREWV, W. 1991. Hospitality today: an introduction. Michigan: The Educational Institute of the American Hotel \& Motel Association. 